\title{
Caracterización de prácticas pedagógicas de personal docente adscrito a liceos del programa de acompañamiento y acceso efectivo a la educación superior (PACE)
}

\author{
Characterization of pedagogical practice of high school teachers participating on the \\ program of assistance and effective access to higher education \\ Impacto das habilidades de compreensão de leitura na aprendizagem escolar: um estudo
realizado em uma comunidade na região metropolitana do Chile
}

Felipe Sepúlveda

Universidad Católica de la Santísima Concepción

Concepción, Chile

fsepulveda@ucsc.cl

(iD http://orcid.org/0000-0001-5703-383X

Edith Calderón

Universidad Católica de la Santísima Concepción

Concepción, Chile

ecalderon@ucsc.cl

(iD) http://orcid.org/0000-0002-9440-5388

María José Espinoza

Universidad Católica de la Santísima Concepción

Concepción, Chile

mjespinoza@ucsc.cl

D https://orcid.org/0000-0001-5850-2722

\author{
Recibido • Received • Recebido: 22 / 06 / 2017 \\ Corregido • Revised • Revisado: 05 / $10 / 2018$ \\ Aceptado • Accepted • Aprovado: 30 / 01 / 2019
}

\begin{abstract}
Resumen: El programa de Acompañamiento y Acceso Efectivo a la Educación Superior (PACE) tiene como objetivo final entregar una oportunidad de continuidad de estudios a nivel superior para estudiantes que asisten a establecimientos educacionales con altos índices de vulnerabilidad social. EI PACE está diseñado para trabajar desde tercer año de enseñanza media con el objetivo de entregar orientación vocacional y apoyar la formación académica. Este estudio describe los resultados de un diagnóstico sobre prácticas pedagógicas en el área de lenguaje y comunicación y matemáticas, desarrollado en 15 liceos de la región del Biobío, Chile. Se realizaron observaciones de clase a 58 docentes con el objetivo de caracterizar la practicas pedagógicas. Los resultados fueros utilizados para trabajar con cada docente en la implementación de nuevas metodologías de enseñanza acordadas entre el equipo de Preparación y Acompañamiento Docente del PACE UCSC y cada docente de aula.
\end{abstract}

Palabras claves: Escuelas eficaces; práctica docente; práctica educativa; mejoramiento profesional; salón de clases. 
doi: http://dx.doi.org/10.15359/ree.23-2.8

URL: http://www.una.ac.cr/educare

CORREO: educare@una.cr

\begin{abstract}
The program called "Assistance and Effective Access to Higher Education" (PACE) aims to provide an opportunity to continue studies at a higher education institution to high school students attending educational establishments with high levels of social vulnerability. The PACE is designed to work with students from eleventh grade, providing them with vocational guidance and supporting their secondary academic formation. This study describes the results of a characterization of pedagogical practices of language arts and mathematics classes, developed in 15 high schools from Biobío region, Chile. The pedagogical practices of 58 participating teachers were characterized throughout in-class observations. The PACE UCSC advisor team and each classroom teacher used the observation results to design an action plan directed to develop effective teaching methodologies.
\end{abstract}

Keywords: Effective schools; teaching practice; educational practice; professional development; classrooms.

Resumo: O programa de Acompanhamento e Acesso Eficaz ao Ensino Superior (PACE) tem como objetivo final proporcionar uma oportunidade de continuidade de estudos a nível superior a estudantes que frequentam estabelecimentos de ensino com altos níveis de vulnerabilidade social. O PACE foi concebido para trabalhar a partir do terceiro ano do ensino médio, com o objetivo de fornecer orientação vocacional e apoiar a formação acadêmica. Este estudo descreve os resultados de um diagnóstico de práticas pedagógicas na área de linguagem, comunicação e matemática, desenvolvida em 15 colégios de ensino médio na região de Biobío, Chile. Foram realizadas observações a 58 professores em suas classes, com o objetivo de caracterizar as práticas pedagógicas. Os resultados foram utilizados para trabalhar com cada professor na elaboração de novas metodologias de ensino, entre a equipe de Preparação e Acompanhamento de Professores do PACE UCSC e cada professor em sala de aula.

Palavras-chave: escolas eficazes; prática docente; prática educativa; aprimoramento profissional; sala de aula.

\title{
Introducción
}

A partir del año 2014 la actual administración del Gobierno de Chile ha impulsado el Programa de Acompañamiento y Acceso Efectivo a la Educación Superior (PACE) que se basa en la convicción de que "los talentos académicos están igualmente distribuidos en la población sin distinguir, por raza o nivel socioeconómico" (Kri, Gil, González, y Lamatta, 2013, p. 8). Así, el propósito fundamental del programa PACE es que "todos los estudiantes, sin excepción, tienen derecho a una educación de calidad que asegure su participación y aprendizaje en los diferentes niveles de enseñanza" (Mineduc, República de Chile, 2015, p. 1), valora el esfuerzo de quienes destacan académicamente en su contexto educativo particular, pese a que aquellos resultados no parezcan significativos respecto de contextos privilegiados.

EIProgramaPACE trabajaconestablecimientos educacionales cuyoíndicedevulnerabilidad escolar (IVE-SINAE) es alto, información recabada a través de una metodología de medición de la condición de vulnerabilidad llevada a cabo por la Junta Nacional de Auxilio Escolar y Becas

Felipe Sepúlveda, Edith Calderón y María José Espinoza

Los artículos de la Revista Electrónica Educare del Centro de Investigación y Docencia en Educación de la Universidad Nacional, Costa Rica, se comparten bajo términos de la Licencia Creative Commons: Reconocimiento, No Comercial, Sin Obra Derivada 3.0 Costa Rica. Las autorizaciones adicionales a las aquí delimitadas se pueden obtener en el correo: educare@una.cr 
(JUNAEB). Para intervenir en estos contextos educativos se trabaja mediante tres estrategias de acompañamiento en la comunidad y una estrategia de acompañamiento y retención en la educación superior: Preparación Académica y Acompañamiento Docente (PAAD), Preparación para la Vida (PPV) y Vinculación entre Comunidades (VEC); Acompañamiento y Retención en la Educación Superior (AES).

El programa PACE UCSC busca contribuir al mejoramiento de las prácticas pedagógicas, en concordancia con el Proyecto de Mejoramiento Educativo (PME) y el Proyecto Escolar Intitucional (PEI) de los liceos particpantes, para favorecer el desarrollo de las competencias (conocimientos, habilidades y actitudes) comprendidas en los objetivos fundamentales y contenidos mínimos obligatorios presentes el marco curricular de la enseñanza media establecido por el Mineduc. Aun cuando el trabajo directo de profesionales de la estrategia PAAD es desarrollado directamente con el profesorado de los establecimientos educacionales (EE), el fin último fue apoyar en la preparación de estudiantes de los establecimientos PACE UCSC, considerando la posibilidad de ingreso y permanencia en el sistema de educación superior.

\section{Objetivo de la investigación}

Tomando en cuenta que el acompañamiento académico tiene como fin potenciar el trabajo realizado por el equipo docente, al atender las necesidades contextuales de los EE a los que pertenecen, es pertinente recabar datos respecto de los procesos pedagógicos -traducidos en métodos, prácticas, estrategias, etc.- que se despliegan en las salas de clase. Por esta razón, se propuso una investigación cuyo fin radicó en la ejecución de un diagnóstico para evidenciar las prácticas pedagógicas del profesorado y, de esta forma, identificar las principales estrategias o metodologías que podrían perfeccionarse por medio de un plan de acompañamiento en el contexto del programa PACE-UCSC. Dicha investigación tuvo como objetivo describir las prácticas académicas más utilizadas, tanto en Lenguaje y Comunicación como en Matemática, por parte de docentes que atienden a tercero medio de establecimientos educacionales de la región del Biobío, adscritos al programa PACE-UCSC.

\section{Marco conceptual}

Conceptos como calidad de enseñanza, efectividad docente y buenas prácticas educativas son permanentemente utilizados en el ámbito educativo. A continuación, se desarrolla una revisión conceptual que aborda estos términos contextualizados con base en la realidad educativa chilena y considerando la observación del aula como una herramienta de recogida de información útil que promueve la reflexión sobre las prácticas docentes en el aula. 
doi: http://dx.doi.org/10.15359/ree.23-2.8

URL: http://www.una.ac.cr/educare

CORREO: educare@una.cr

\section{El marco regulatorio para el personal docente chileno basado en el sistema de aseguramiento de la calidad de la educación escolar}

En los últimos 15 años, gran parte de las reformas educacionales de Chile se han enfocado en avanzar hacia el mejoramiento de la calidad de la educación financiada con fondos públicos. Esto se ha traducido en la creación de nuevas instituciones gubernamentales (Agencia de la Calidad la Educación y Superintendencia de Educación) y normativas (Marco para la Buena Enseñanza y Estándares Indicativos de Desempeño) enmarcadas en el diseño e implementación de un sistema de aseguramiento de la calidad de la educación.

El Marco para la Buena Enseñanza (MBE) es una guía orientadora que "establece lo que los docentes chilenos deben conocer, saber hacer y ponderar para determinar cuán bien lo hace cada uno en el aula y en la escuela" (Mineduc, República de Chile, 2008, p. 5). Esta herramienta constituye la base de todos los instrumentos del Sistema de Evaluación Docente (portafolio, pauta de autoevaluación, entrevista por un sujeto evaluador par e informes de referencia de terceras personas), pues se construyen de acuerdo con sus dominios, criterios y descriptores.

En tanto, los Estándares Indicativos de Desempeño (EID) "son referentes que orientan la Evaluación Indicativa de Desempeño a cargo de la Agencia de Calidad y que, a la vez, entregan orientaciones a los establecimientos y sus sostenedores para mejorar los procesos de gestión institucional" (Mineduc, República de Chile, 2014, p. 17). Dicho marco normativo "define una institucionalidad que aborda el problema de la calidad educativa desde una perspectiva sistémica, y busca un equilibrio entre la autonomía de los sostenedores, la entrega de apoyo y la exigencia de rendición de cuentas" (Mineduc, República de Chile, 2014, p. 9). Al respecto, además, tiene como objetivo servir de base para la evaluación indicativa de desempeño a cargo de la Agencia de Calidad, cuyo interés es considerar no solo lo planteado en dicha herramienta, sino también el contexto del establecimiento educacional, sus resultados educativos y el grado de cumplimiento de los estándares de aprendizaje y de otros indicadores de calidad. Por esta razón, en dicho proceso cobran relevancia como antecedentes el proyecto educativo institucional y los resultados de la autoevaluación del establecimiento.

\section{Asociaciones entre la formación pedagógica y la efectividad de la enseñanza}

Existe una amplia evidencia que apunta hacia la importancia del profesorado en el mejoramiento del desempeño académico del estudiantado en el corto y largo plazo, como también en relación con la enorme variabilidad en su efectividad de enseñanza. Diversas investigaciones indican que docentes con determinadas características pueden tener un poderoso efecto en el desarrollo de habilidades cognitivas y metacognitivas del estudiantado, incluso cuando este efecto no es perceptible a nivel del colegio (Caro, Lenkeit, y Kyriakides, 2016). 
Sin duda, la formación profesional y la formación en servicio del personal docente son elementos fundamentales para el desempeño en el aula. En este contexto, Hanushek (1997) desarrolla una revisión de antecedentes con el propósito de establecer relaciones entre ciertas habilidades y caracteristicas docentes junto con su efectividad para el desarrollo de aprendizajes en el aula. Este tipo de estudios no ha sido concluyente con respecto a la relación entre los años de estudio o preparación académica del profesorado con los logros de aprendizajes de sus estudiantes (Hanushek, 1997). A pesar de esto, un estudio desarrollado por Gore et al. (2015) evidencia un impacto en la calidad de la enseñanza impartida por parte de docentes participantes en un programa de capacitación docente que integra el desarrollo de comunidades profesionales de aprendizaje y las rondas de instrucción en un marco definido sobre la enseñanza de calidad (Quality Teaching Framework). Así mismo, se ha identificado que la calidad de la experiencia en los primeros años de ejercicio de la profesión es un factor relevante que explica el desempeño docente en el aula (Rockoff, 2004) y de la mayor efectividad de quienes hayan sido buenos o buenas aprendices en su etapa escolar o que hayan obtenido buenos resultados en las pruebas de entrada a la universidad (Goldhaber, 2008).

\section{Características y prácticas docentes comúnmente asociadas con la enseñanza efectiva}

Existen variados factores y dinámicas de aula que son reconocidos como elementos clave que definen la efectividad del personal docente dentro de los que destacan el compromiso docente, clima de aula, preparación y organización, estrategias didácticas, gestión del tiempo, evaluación y retroalimentación de aprendizajes, y la atención a la diversidad, según lo identificado por Murillo, Hernández-Castilla y Martínez-Garrido, (2016). Igualmente se indica que una profesora o profesor efectivo se caracteriza por su habilidad para usar estrategias y técnicas didácticas y del uso eficiente de las tecnologías y los recursos de la información (Avanzini, 2004). En conjunto con lo anterior, el profesorado ha de conocer y saber aplicar los criterios para seleccionar la estrategia o técnica más adecuada para su curso. De esta forma, el profesorado efectivo cuando planifica su clase es capaz de tomar decisiones de manera consciente y reflexiva en cuanto a las técnicas y actividades que puede utilizar para llegar a lograr aprendizajes de calidad.

De acuerdo con los elementos que caracterizan al personal docente efectivo, se comprende que los procesos pedagógicos que ocurren al interior de las prácticas pedagógicas pueden ser definidos como actos situados en un sistema abierto y complejo, como lo es la acción pedagógica que busca lograr aprendizaje en el estudiantado. Así, el comportamiento del profesorado y las dinámicas que acontecen en el interior del aula son considerados algunos de los factores más significativos para explicar los resultados académicos y las habilidades metacognitivas del estudiantado en el aula (Caro et al., 2016). En este contexto, las prácticas pedagógicas aparecen como un espacio privilegiado en el que se encuentran: 
doi: http://dx.doi.org/10.15359/ree.23-2.8

URL: http://www.una.ac.cr/educare

CORREO: educare@una.cr

acciones de enseñanza y aprendizaje, donde se reúnen profesor/a y estudiantes y estructuran relaciones de diversa naturaleza: epistemológicas (a propósito del conocimiento, los saberes a enseñar y aprender), didácticas (a propósito de las estrategias de enseñanza que se implementan) y sociales (donde en el contexto de una comunidad participan sujetos individuales y actores, en tanto son miembros de diferentes grupos etarios, socioeconómicos y culturales). (Latorre, 2004, p. 81)

Con el propósito de relacionar las prácticas docentes con la enseñanza efectiva, resulta interesante acudir a estudios cuyos resultados han demostrado que el estudiantado competente parece saber mucho más sobre las estrategias cognitivas generales -cómo y cuándo aplicarlas- que las personas menos competentes, tal es el caso del desarrollo de la creatividad como una habilidad de nivel superior. Hargrove (2011) profundiza en tres elementos que se deben considerar en las prácticas efectivas, en primer lugar, que las practicas docentes han de involucrar la enseñanza explícita de estrategias de aprendizaje; en segundo lugar, que el estudiantado aprenda sobre el pensamiento metacognitivo y la relación entre cognición y metacognición y, tercero, que se produzca la reflexión del estudiantado sobre sus modelos mentales que se ejecutan mientras aprenden.

Otro elemento importante en la implementación de las prácticas efectivas es la interacción que se ha de producir entre docente y estudiante, requisito esencial que favorece el ajuste entre la actividad de aprendizaje y las necesidades del alumnado, tal como ocurre en los estudios referidos a la indagación en ciencias como habilidad cognitiva, donde se demostró que el rol del profesorado involucra una cierta flexibilidad para la unión entre lo afectivo con lo cognitivo, todo ello acompañado de la retroalimentación y el monitoreo permanente por la parte docente (González-Weil et al., 2012) hasta lograr el aprendizaje de sus estudiantes.

Debido a la necesidad de crear experiencias de aprendizaje para todo el estudiantado, se propone como estrategia el desarrollo de acciones pedagógicas compartidas entre el profesorado, la co-enseñanza, la cual se fundamenta en que el aprendizaje efectivo resulta de los esfuerzos colaborativos entre el profesorado. Su implementación se traduce en el establecimiento de metas recíprocas y de compartir los recursos para favorecer el éxito de todo el estudiantado (McLeskey, Waldron, Spooner y Algozzine, 2014). A través del equipo colaborativo en la co- enseñanza, se logran establecer los estándares comunes de contenidos para la totalidad del estudiantado y el uso de la evaluación auténtica.

Así, se debe entender que "el aprendizaje es un proceso multifactorial que demanda pensarlo y repensarlo, a fin de que el alumnado logre disfrutarlo e interiorizarlo mientras se desarrolla de manera integral en los espacios escolares" (Castro-Pérez y Morales-Ramírez, 2015, p. 28). Al docente o a la docente que quiere llegar a ser una profesora o profesor efectivo, le implicará modificar sus prácticas e incorporar diversas estrategias y técnicas orientadas a establecer compromisos y responsabilidades por parte del estudiantado a través de un proceso de reflexión permanente sobre los logros de aprendizaje y de la forma en que se alcanzaron. 


\section{Observaciones de aula como una herramienta de reflexión del desempeño docente}

El análisis de las prácticas pedagógicas en el aula necesariamente requiere un proceso de recogida de información que dé cuenta de las interacciones docente-estudiante y las estrategias utilizadas para facilitar los procesos de enseñanza-aprendizaje. En general, las observaciones de clase buscan registrar las prácticas instruccionales del personal docente y su habilidad para estructurar y mantener un ambiente de clase altamente funcional (Steinberg y Garrett, 2016). Las observaciones de aula constituyen un mecanismo que permite relacionar los métodos desplegados en la sala de clases con el progreso académico del estudiantado. Junto con ello, se provee un feedback formativo para los docentes y las docentes, cuyo fin es contribuir y encauzar los esfuerzos de mejora (Martinez, Taut y Schaaf, 2016). Comúnmente, las observaciones de aula son utilizadas como un instrumento para evaluar el desempeño del personal docente en el aula. Cohen y Goldhaber (2016) indican que este tipo de instrumento presenta una alta validez por parte del equipo docente, puesto que es él mismo quien logra observar su propia práctica de enseñanza. En este sentido, las observaciones de aula son una fuente directa de retroalimentación profesional necesaria para el mejoramiento a través de la comunicación con otros individuos con respecto a lo que pasa en las salas de clase (Liang, 2015). Si bien las observaciones de aula suelen ser utilizadas como un instrumento de evaluación de desempeño, en el marco de la implementación del acompañamiento docente, vinculado al programa PACE, esta herramienta posibilita el levantamiento de información relevante para la identificación de las prioridades de apoyo que requieren cada docente del programa. En este sentido, Kim y Silver (2016) destacan la utilidad de las observaciones de aula como factor relevante para promover procesos de reflexión entre docentes nóveles y sus mentores o mentoras.

\section{Metodología}

Para efectos del trabajo que aquí se presenta, se focalizará en lo desarrollado por la estrategia PAAD, equipo que, a través de la Universidad Católica de la Santísima Concepción (UCSC), ha trabajado desde el 2015 con 15 establecimientos educacionales con alto IVE, suma que se acrecentó el 2016, ascendiendo a 22 los liceos objetivo. Así, en esta investigación se informan los resultados relativos a las prácticas pedagógicas más comunes de los docentes y las docentes de Lenguaje y Comunicación y Matemática y que participaron en el proceso de acompañamiento y preparación académica.

De acuerdo con los requerimientos del Ministerio de Educación, se construyó un plan de trabajo tendiente al acompañamiento académico-pedagógico de docentes de Lenguaje y Comunicación junto con Matemática que trabajaban en $3^{\circ}$ y $4^{\circ}$ medio de dichos establecimientos. 
doi: http://dx.doi.org/10.15359/ree.23-2.8

URL: http://www.una.ac.cr/educare

CORREO: educare@una.cr

El Programa de Acompañamiento Docente PACE UCSC se llevó a cabo en cinco etapas:

1. Observación de aula: Aplicación del instrumento de Observación de aula, retroalimentación positiva a cada docente de aula, sistematización de la información y confección del diagnóstico.

2. Plan de acción: Reflexión sobre prácticas docentes junto con cada docente de aula, consenso del plan de acción e identificación de dos o tres áreas de crecimiento en sus prácticas pedagógicas.

3. Ejecución del plan de acción: Talleres disciplinares y de actualización, validación académica, demostración de aula, apoyo pedagógico.

4. Segunda observación de aula: Aplicación del instrumento de Observación de aula, retroalimentación positiva a cada docente de aula, sistematización de la información y verificación de mejoras basadas en plan de acción

5. Retroalimentación final: Confección de informe final por establecimiento, identificación de patrones generalizados en todos los establecimientos PACE UCSC

\section{Participantes}

Las observaciones se realizaron en 15 establecimientos educacionales de administración pública (Liceos Municipales), correspondientes a cuatro provincias de la VIII región del Biobío, Chile. Los liceos participantes del estudio formaron parte del Programa PACE implementado por la Universidad Católica de la Santísima Concepción en el año 2015 (PACE UCSC), los cuales fueron seleccionados por el Ministerio de Educación Chileno conforme al índice de vulnerabilidad (IVESINAE) del estudiantado que forma parte de la matrícula del establecimiento. En su mayoría, los liceos incluidos en el estudio imparten enseñanza técnico-profesional (14 de 15), cuatro de ellos se localizan en área urbana, tres en sectores suburbanos y cuatro pueden ser considerados como rurales.

Uno de los objetivos del programa PACE se relaciona con la implementación de un plan de acompañamiento dirigido al personal docente de las áreas de Matemática y Lenguaje que enseñan en los cursos correspondientes a los dos últimos años previos a la enseñanza superior. Por esta razón, las observaciones fueron dirigidas a los 58 docentes de estas áreas de enseñanza que se encontraban trabajando en cada liceo participante al momento del estudio (Tabla 1), donde el número de estudiantes promedio por aula observada fue de 19 ( $D E=7.1)$. 
Tabla 1: Número de docentes que se observaron

\begin{tabular}{lccc}
\hline & \multicolumn{3}{c}{ Curso } \\
\cline { 2 - 4 } Provincia & Lenguaje y Comunicación & Matemática & Total \\
\hline Concepción & 8 & 5 & 13 \\
Arauco & 15 & 13 & 28 \\
Nuble & 2 & 7 & 9 \\
Bio-bío & 2 & 6 & 8 \\
\hline Total & 27 & 31 & 58 \\
\hline
\end{tabular}

Nota: Elaboración propia.

\section{Instrumento utilizado para la observación de aula}

Los registros de las observaciones de clase fueron sistematizados con base en los estándares indicativos de desempeño definidos como referentes para establecer metas y acciones en la elaboración de planes de mejoramiento por parte de la Agencia de Calidad de Educación chilena (Mineduc, República de Chile, 2014). La pauta de observación de aula fue construida a partir del instrumento diseñado por el centro de investigación The Charles A. Dana Center (2011) de la Universidad de Texas, que centra los registros en la planificación de la clase, lo que hace el estudiantado y los maestros y las maestras durante el desarrollo de esta misma. Este instrumento fue seleccionado por su alta validez de constructo, además de que ha sido extensamente utilizado en el marco de programas de mejoramiento continuo desplegados por instituciones educativas correspondientes al sistema público de Texas. Adicionalmente, el instrumento permite registrar variables nominales en categorías que son fácilmente identificables por los observadores de aula. De esta manera, se construyó una pauta de observación que incluía tres estándares de la subdimensión "Enseñanza y Aprendizaje en el Aula" dirigidos a los procedimientos y prácticas que implementan los profesores en sus clases para asegurar el logro de los objetivos de aprendizaje (Tabla 2). Los estándares seleccionados se relacionan con el uso de estrategias efectivas de enseñanza-aprendizaje y manejo de la clase como elementos claves para lograr el aprendizaje del estudiantado.

\section{Procedimiento y análisis}

Las visitas de aula para observar las clases se realizaron en un intervalo de ocho semanas, entre el 3 de septiembre y el 26 de octubre de 2015. Encargado de dicha tarea fue el equipo docente facilitador perteneciente al Equipo de Preparación Académica y Acompañamiento Docente del PACE-UCSC. Cada profesional asistió a las clases, previas reuniones en el marco 
doi: http://dx.doi.org/10.15359/ree.23-2.8

URL: http://www.una.ac.cr/educare

CORREO: educare@una.cr

del plan de acompañamiento docente con el profesorado que participaría en el estudio. De esta forma, equipo facilitador y profesorado participantes acordaron en conjunto el momento propicio para desarrollar la observación. Estas visitas se desarrollaron por un periodo completo de clases (90 minutos) y no existió interacción con el profesor o profesora de aula durante el desarrollo de la clase observada. Una vez terminada dicha clase se entregó una retroalimentación (positiva) a cada docente y se programó una nueva reunión para la revisión en profundidad de los elementos observados con el fin de acordar los elementos que incluirían en un plan de acción y acompañamiento acorde con el curso y las características de cada docente que se acompañó.

Tabla 2: Estándares e indicadores utilizados para valorar su implementación en el aula

\begin{tabular}{|c|c|c|}
\hline Estándar & Indicador & Categorías \\
\hline \multirow{4}{*}{$\begin{array}{l}\text { 5.1 El profesorado imparte las clases } \\
\text { en función de los objetivos de } \\
\text { aprendizaje estipulados en las } \\
\text { bases curriculares }\end{array}$} & ¿Existe una planificación previa de la clase?: & $\begin{array}{l}\text {-Existe } \\
\text {-No Existe }\end{array}$ \\
\hline & $\begin{array}{l}\text { ¿La clase observada se ajusta a lo declarado } \\
\text { en la planificación? }\end{array}$ & $\begin{array}{l}\text {-Sí } \\
\text {-No }\end{array}$ \\
\hline & $\begin{array}{l}\text { ¿El objetivo de la clase se encuentra de } \\
\text { acuerdo con lo esperado en tercero medio? }\end{array}$ & $\begin{array}{l}\text {-Sí } \\
\text {-No }\end{array}$ \\
\hline & $\begin{array}{l}\text { ¿El objetivo de aprendizaje es comprendido } \\
\text { por el estudiantado? }\end{array}$ & $\begin{array}{l}\text { Porcentaje de estudiantes capaces de verbalizar } \\
\text { adecuadamente el objetivo de la clase }\end{array}$ \\
\hline \multirow[t]{3}{*}{$\begin{array}{l}\text { 5.2 El profesorado conduce las } \\
\text { clases con claridad, rigurosidad } \\
\text { conceptual, dinamismo e interés. }\end{array}$} & $\begin{array}{l}\text { Identifica las prácticas de instrucción } \\
\text { desarrolladas en el aula }\end{array}$ & $\begin{array}{l}\text {-Exposición } \\
\text {-Discusión } \\
\text {-Evaluación formal } \\
\text {-Modelamiento } \\
\text { de Aprendizaje } \\
\text {-Presentación } \\
\text {-Entrega de direcciones/instrucciones } \\
\text {-Experiencias directas con material educativo } \\
\text {-Preguntas/Respuestas } \\
\text {-Otra }\end{array}$ \\
\hline & $\begin{array}{l}\text { Determina el nivel(es) del trabajo requerido } \\
\text { por el estudiantado }\end{array}$ & $\begin{array}{l}\text {-Conocimiento } \\
\text {-Compresión } \\
\text {-Aplicación } \\
\text {-Análisis } \\
\text {-Síntesis } \\
\text {-Evaluación }\end{array}$ \\
\hline & $\begin{array}{l}\text { Identifica las estrategias de instrucción } \\
\text { utilizadas en el aula }\end{array}$ & $\begin{array}{l}\text {-Identificar similitudes y diferencias } \\
\text {-Aprendizaje cooperativo } \\
\text {-Toma de apuntes y síntesis de información } \\
\text {-Trabajo colaborativo } \\
\text {-Generar y verificar hipótesis } \\
\text {-Representaciones no verbales o para verbales } \\
\text {-Definir un objetivo de aprendizaje y entrega de } \\
\text { retroalimentación del aprendizaje } \\
\text {-Estimular el esfuerzo y entrega de reconocimiento } \\
\text {-Organizadores gráficos } \\
\text {-Ninguna de las anteriores } \\
\text {-Otra }\end{array}$ \\
\hline
\end{tabular}

continúa 


\begin{tabular}{|c|c|c|}
\hline \multirow[t]{3}{*}{$\begin{array}{l}\text { 5.3 El profesorado utiliza estrategias } \\
\text { efectivas de enseñanza- } \\
\text { aprendizaje en el aula. }\end{array}$} & $\begin{array}{l}\text { Identifica el patrón de agrupamiento } \\
\text { de estudiantes durante el tiempo de } \\
\text { instrucción: }\end{array}$ & $\begin{array}{l}\text {-Toda la clase } \\
\text {-Pequeños grupos } \\
\text {-En parejas } \\
\text {-Individual }\end{array}$ \\
\hline & $\begin{array}{l}\text { Identifica los materiales utilizados para el } \\
\text { aprendizaje }\end{array}$ & $\begin{array}{l}\text {-Hoja de actividad } \\
\text {-Manipulables, material o modelos concretos } \\
\text {-Material impreso (libros, revistas) } \\
\text {-Texto escolar } \\
\text {-Material creado por el estudiante } \\
\text {-Sitios WEB } \\
\text {-Tecnología/Software } \\
\text {-Otro }\end{array}$ \\
\hline & $\begin{array}{l}\text { Identifica de qué manera el estudiantado } \\
\text { adquiere, entiende y comunica el } \\
\text { conocimiento del contenido de la lección }\end{array}$ & $\begin{array}{l}\text {-Escuchando } \\
\text {-Leyendo } \\
\text {-Hablando } \\
\text {-Escribiendo }\end{array}$ \\
\hline
\end{tabular}

Nota: Adaptado a partir la pauta de observacion Classroom Walkthrough for Continuous Improvement: Strengthening Teaching and Learning, del centro de investigación educativa The University of Texas at Austin (The Charles A. Dana Center, 2011).

El personal docente facilitador del programa PACE-UCSC fue instruido con respecto a la operacionalización del instrumento de evaluación utilizado, puntualizando algunos casos concretos para la valoración de cada ítem de la pauta de observación. Adicionalmente, se acordó un protocolo de observación de aula que detallaba los pasos a seguir previamente, durante y una vez concluida la visita a la sala de clases. Para la sistematización y el análisis de los datos, se utilizaron tablas de frecuencia de ocurrencia que consideraban cada variable sondeada por la pauta antes mencionada.

\section{Resultados}

Lainformación recogida a partir delas observaciones de clasesfueorganizada dandocuenta de la frecuencia de ocurrencia de los indicadores observados en las asignaturas de Matemática y Lenguaje y Comunicación. La información se presenta considerando los diferentes estándares indicativos de desempeño asociados a los elementos observados en las salas de clase dirigidas por docentes participantes del programa PACE UCSC. Los datos fueron analizados utilizando un análisis no paramétrico de chi-cuadrado para evaluar diferencias significativas con respecto a las categorías observadas. De esta manera, se determinó si los indicadores observados bajo cada estándar presentaban una distribución homogénea o heterogénea, considerando las dos asignaturas antes mencionadas.

\section{El profesorado imparte las clases en función de los objetivos de aprendizaje estipulados en las bases curriculares}

Las observaciones registradas referidas al estándar 5.1 de la sección enseñanza y aprendizaje en el aula de los Estándares Indicativos de Desempeño (Mineduc, República de Chile, 2014) referidos a si los docentes y las docentes imparten clases alineadas a los objetivos de 
doi: http://dx.doi.org/10.15359/ree.23-2.8

URL: http://www.una.ac.cr/educare

CORREO: educare@una.cr

aprendizaje estipulados en las Bases Curriculares fueron valoradas mediante cuatro indicadores que incluían el registro de las planificaciones del equipo docente y las observaciones de aula. Se realizó un análisis de chi-cuadrado para verificar la homogeneidad de la frecuencia registrada para cada indicador, considerando cada asignatura observada (Tabla 3). En este caso, no se registraron asociaciones significativas entre las variables analizadas bajo este estándar, según el siguiente detalle: a) presencia de una planificación de la clase para cada curso $p=$ $0.760(1,58$, chi cuadrado $=0.094, \mathrm{~V}$ de Cramer $=0.040)$; b) planificación informada ajustada al desarrollo de la clase $p=0.994(2,58$, chi cuadrado $=0.011, \mathrm{~V}$ de Cramer $=0.014)$; c) Objetivos informados alineados con el nivel de tercero medio $p=0,960(1,58$, chi cuadrado $=0.002, \mathrm{~V}$ de Cramer $=0.007)$; d) Porcentaje de estudiantes que entienden el objetivo de la clase en que están participando $p=0.120(4,58$, chi cuadrado $=7,316$, V de Cramer $=0.358)$.

Tabla 3: Frecuencia de ocurrencia en categorías definidas para los indicadores de implementación del estándar 5.1

\begin{tabular}{|c|c|c|c|}
\hline & \multicolumn{3}{|c|}{ Asignatura } \\
\hline & Lenguaje y Comunicación & Matemática & Total \\
\hline \multicolumn{4}{|l|}{ Planificación de la Clase } \\
\hline Existe & $44,8 \%$ & $43,1 \%$ & $87,9 \%$ \\
\hline No existe & $0,0 \%$ & $0,0 \%$ & $0 \%$ \\
\hline No se puede determinar & $6,9 \%$ & $5,2 \%$ & $12,1 \%$ \\
\hline \multicolumn{4}{|c|}{ Clase se ajusta a lo planificado } \\
\hline Sí & $44,8 \%$ & $41,4 \%$ & $86,2 \%$ \\
\hline No & $1,7 \%$ & $1,7 \%$ & $3,4 \%$ \\
\hline No determinado & $5,2 \%$ & $5,2 \%$ & $10,3 \%$ \\
\hline \multicolumn{4}{|c|}{ Objetivo alineado con el nivel del curso } \\
\hline Sí & $50,0 \%$ & $46,6 \%$ & $96,6 \%$ \\
\hline No & $1,7 \%$ & $1,7 \%$ & $3,4 \%$ \\
\hline \multicolumn{4}{|c|}{ Porcentaje de estudiantes que comprenden el objetivo de la clase } \\
\hline $100 \%$ & $33,3 \%$ & $21,1 \%$ & $54,4 \%$ \\
\hline $80 \%$ & $12,3 \%$ & $5,3 \%$ & $17,5 \%$ \\
\hline $60 \%$ & $5,3 \%$ & $14,0 \%$ & $19,3 \%$ \\
\hline $40 \%$ & $1,8 \%$ & $5,3 \%$ & $7,0 \%$ \\
\hline $20 \%$ & $0,0 \%$ & $0,0 \%$ & $0 \%$ \\
\hline $0 \%$ & $0,0 \%$ & $1,8 \%$ & $1,8 \%$ \\
\hline
\end{tabular}

Nota: Elaboración propia. 
Los resultados sugieren que, tanto los docentes y las docentes de los cursos de Lenguaje y Comunicación como Matemática se encuentran acostumbrados a desarrollar e implementar una adecuada planificación de sus clases conforme al nivel y contenidos pertinentes al curso observado. Un elemento que llama la atención en las clases observadas es la proporción de estudiantes que comprenden el objetivo de la clase en que están participando. Así, solo un poco más de la mitad $(54,4 \%)$ del estudiantado consultado fue capaz de verbalizar adecuadamente los objetivos de la clase en que se encontraban participando.

\section{El profesorado conduce las clases con claridad, rigurosidad conceptual, dinamismo e interés}

Las observaciones registradas referidas al estándar 5.2 de la sección enseñanza y aprendizaje en el aula de los Estándares Indicativos de Desempeño (Mineduc, República de Chile, 2014), los que se enfocan en el desempeño del personal docente durante el desarrollo de la clase, enfatizando la manera en que explica, demuestra, entrega instrucciones y ejemplos, utiliza elementos de apoyo visual y facilita la compresión de conocimientos, fue valorada mediante tres indicadores que incluyeron el registro de las prácticas de instrucción desarrollada por cada docente, nivel de habilidades cognitivas requeridas por el estudiantado para ejecutar la actividad planteada en la clase y la estrategia de instrucción utilizada por el personal docente para facilitar la adquisición del objetivo de aprendizaje planificado en la clase (Tabla 4).

Se detectaron diferencias significativas entre la variedad de prácticas de instrucción observadas en las clases de Matemática y Lenguaje y Comunicación, $p<0.001(8,118$, chicuadrado $=25,668, \mathrm{~V}$ de Cramer $=0.466$ ). En este sentido, se aprecia que las prácticas más utilizadas son las clases de tipo expositiva, registrada en un 39\% de las clases observadas, seguido por la utilización de preguntas y respuestas (18,6\% de los casos). En el caso de Lenguaje y Comunicación, se aprecia la utilización de una mayor variedad de estrategias de instrucción en comparación con las clases de Matemática.

Igualmente se detectaron diferencias significativas entre el nivel de habilidades cognitivas de las actividades desarrolladas en las clases de Matemática y Lenguaje y Comunicación, $\mathrm{p}$ $=0.039(4,114$, chi-cuadrado $=10,077, \mathrm{~V}$ de Cramer $=0.297)$. Se aprecia que las actividades asociadas a la adquisición de conocimiento (36\%), comprensión (25\%) y aplicación (23\%) son las más utilizadas en las clases observadas. Actividades asociadas a una mayor demanda cognitiva (análisis, síntesis y evaluación de información) practicamente no son utilizadas en las aulas observadas. En este sentido, cabe destacar que las clases de Matemática observadas se basan principalmente en actividades que requieren el nivel más bajo de demanda cognitiva (desarrollo de conocimientos) de acuerdo a la taxonomía de Bloom. 
doi: http://dx.doi.org/10.15359/ree.23-2.8

URL: http://www.una.ac.cr/educare

CORREO: educare@una.cr

Tabla 4: Frecuencia de ocurrencia en categorías definidas para los indicadores de implementación del estándar 5.2

\begin{tabular}{|c|c|c|c|}
\hline & \multicolumn{3}{|c|}{ Asignatura } \\
\hline & Lenguaje y Comunicación & Matemática & Total \\
\hline \multicolumn{4}{|l|}{ Prácticas de Instrucción* } \\
\hline Exposición & $20,3 \%$ & $18,6 \%$ & $39,0 \%$ \\
\hline Discusión & $11,9 \%$ & $0,8 \%$ & $12,7 \%$ \\
\hline Evaluación formal & $0,0 \%$ & $0,8 \%$ & $0,8 \%$ \\
\hline Modelamiento & $3,4 \%$ & $0,8 \%$ & $4,2 \%$ \\
\hline Centro de aprendizaje & $0,0 \%$ & $0,0 \%$ & $0,0 \%$ \\
\hline Presentación & $8,5 \%$ & $0,8 \%$ & $9,3 \%$ \\
\hline Entrega de direcciones/instrucciones & $7,6 \%$ & $0,0 \%$ & $7,6 \%$ \\
\hline Experiencias directas con material educativo & $5,1 \%$ & $0,0 \%$ & $5,1 \%$ \\
\hline Preguntas / Respuestas & $10,2 \%$ & $8,5 \%$ & $18,6 \%$ \\
\hline Otras & $0,0 \%$ & $0,0 \%$ & $0,0 \%$ \\
\hline \multicolumn{4}{|l|}{ Nivel de habilidades cognitivas requerida en la actividad ** } \\
\hline Conocimiento & $20,2 \%$ & $15,8 \%$ & $36,0 \%$ \\
\hline Compresión & $20,2 \%$ & $5,3 \%$ & $25,4 \%$ \\
\hline Aplicación & $16,7 \%$ & $7,0 \%$ & $23,7 \%$ \\
\hline Análisis & $7,0 \%$ & $0,9 \%$ & $7,9 \%$ \\
\hline Síntesis & $0,0 \%$ & $0,0 \%$ & $0,0 \%$ \\
\hline Evaluación & $7,0 \%$ & $0,0 \%$ & $7,0 \%$ \\
\hline \multicolumn{4}{|l|}{ Estrategias de Instrucción $* *$} \\
\hline Identificación de similitudes y diferencias & $5,2 \%$ & $8,6 \%$ & $13,8 \%$ \\
\hline Aprendizaje cooperativo & $12,9 \%$ & $0,9 \%$ & $13,8 \%$ \\
\hline Toma de apuntes y síntesis de información & $13,8 \%$ & $0,0 \%$ & $13,8 \%$ \\
\hline Trabajo cooperativo & $9,5 \%$ & $0,0 \%$ & $9,5 \%$ \\
\hline Generar y verificar hipótesis & $5,2 \%$ & $0,9 \%$ & $6,0 \%$ \\
\hline Representaciones verbales o para-verbales & $6,0 \%$ & $0,0 \%$ & $6,0 \%$ \\
\hline Definir objetivo de aprendizaje y entrega de retroalimentación & $10,3 \%$ & $12,1 \%$ & $22,4 \%$ \\
\hline Estimular el esfuerzo y entrega de reconocimiento & $3,4 \%$ & $0,0 \%$ & $3,4 \%$ \\
\hline Organizadores gráficos & $2,6 \%$ & $0,0 \%$ & $2,6 \%$ \\
\hline Otras estrategias & $5,2 \%$ & $3,4 \%$ & $8,6 \%$ \\
\hline
\end{tabular}

*Valor de $\mathrm{p}<.05 ;{ }^{* *}$ valor de $\mathrm{p}<.001$

Nota: Elaboración propia. 
Por último, se detectaron diferencias significativas entre las estrategias de instrucción utilizadas en las clases de Matemática y Lenguaje y Comunicación, $p<0.001$ (4,116, chi-cuadrado $=40,865, V$ de Cramer $=0.594$ ). En esta categoría se observa que las estrategias de aula utilizadas en las asignaturas de Lenguaje y Comunicación y Matemática son muy diferentes. En el caso de Matemática, la gran mayoría de las clases observadas basaban la instrucción en la definición del objetivo a desarrollar en la clase y la entrega de retroalimentación de trabajo desarrollado, junto con la identificación de similitudes y diferencias en el contenido de la clase. En el caso de las clases de Lenguaje y Comunicación, se observó la utilización de una mayor variedad de estrategias de instrucción, destacando la utilización de aprendizaje cooperativo y la toma de apuntes para sintetizar la información presentada durante la clase.

\section{El profesorado utiliza estrategias efectivas de enseñanza-aprendizaje en el aula}

Las observaciones registradas referidas al estándar 5.3 de la sección enseñanza y aprendizaje en el aula de los Estándares Indicativos de Desempeño (Mineduc, República de Chile, 2014), los que se enfocan principalmente en las prácticas de instrucción utilizadas por el personal docente para promover que el estudiantado practique y aplique las habilidades incluidas en la clase, fue valorada mediante tres indicadores que incluyeron el patrón de agrupamiento del estudiantado al momento de interactuar con el contenido de la lección, los materiales de instrucción utilizados por el estudiantado para interactuar con el contenido de la lección y las estrategias utilizadas para que el estudiantado adquiera, comprenda y comunique el contenido incluido en la lección (Tabla 5).

Se detectaron diferencias significativas entre el patrón de agrupamiento utilizado en las clases de Matemática y Lenguaje y Comunicación, $p=0.003$ (3,73, chi-cuadrado $=13,790, \mathrm{~V}$ de Cramer $=0.435$ ). En este sentido, se aprecia que las prácticas más utilizada son las clases de tipo expositiva (especialmente en las clases de Lenguaje y Comunicación), registrada en un $47,9 \%$ de las clases observadas, seguido por el desarrollo del trabajo de manera individual observado en el $27,4 \%$ de las clases observadas (registrado principalmente en las clases de Matemática). Los resultados muestran una evidente tendencia hacia el desarrollo de una clase guiada principalmente por el profesorado y que promueve el trabajo de manera individual.

Igualmente se detectaron diferencias significativas entre el material de instrucción mayormente utilizado en las clases de Matemática y Lenguaje y Comunicación, p $=0.002$ (7,82, chi-cuadrado $=22,997, \mathrm{~V}$ de Cramer $=0.529)$. En ambas asignaturas se observa la tendencia a utilizar hojas de actividades como el principal recurso para interactuar con el contenido de la clase (31.7\%). Sin embargo, se aprecia que las clases de Lenguaje y Comunicación tienden a utilizar una mayor variedad de recursos pedagógicos en comparación con Matemática. Es importante destacar que se aprecia una tendencia a utilizar tecnologías y software para apoyar la instrucción en el caso de Matemática (observado en un 8,5 \% de las clases visitadas), recurso que casi no se utiliza en las clases de Lenguaje y Comunicación. 
doi: http://dx.doi.org/10.15359/ree.23-2.8

URL: http://www.una.ac.cr/educare

CORREO: educare@una.cr

Por último, no se detectaron diferencias significativas entre las estrategias de aula utilizadas para la adquisición, compresión y comunicación del contenido de las clases de Matemática y Lenguaje y Comunicación, $\mathrm{p}=0.114(3,139$, chi cuadrado $=5,956, \mathrm{~V}$ de Cramer $=0.207)$. Se observan patrones similares en ambas asignaturas observadas, donde predomina el desarrollo de clases en las que el estudiantado debe escuchar el contenido presentado por el personal docente (37.4\%), situación que es muy clara en el caso de las clases de Matemática. En lenguaje se aprecia un mayor balance entre las cuatro competencias asociadas a este indicador.

Tabla 5: Frecuencia de ocurrencia en categorías definidas para los indicadores de implementación del estándar 5.3

\begin{tabular}{|c|c|c|c|}
\hline & \multicolumn{3}{|c|}{ Asignatura } \\
\hline & Lenguaje y Comunicación & Matemática & Total \\
\hline \multicolumn{4}{|l|}{ Patrón de agrupamiento de estudiantes** } \\
\hline Toda la clase & $35,6 \%$ & $12,3 \%$ & $47,9 \%$ \\
\hline Pequeños grupos & $5,5 \%$ & $1,4 \%$ & $6,8 \%$ \\
\hline En parejas & $9,6 \%$ & $8,2 \%$ & $17,8 \%$ \\
\hline Individual & $6,8 \%$ & $20,5 \%$ & $27,4 \%$ \\
\hline \multicolumn{4}{|l|}{ Material de instrucción utilizado** } \\
\hline Hoja de actividades & $15,9 \%$ & $15,9 \%$ & $31,7 \%$ \\
\hline Manipulables, material o modelos concretos & $3,7 \%$ & $2,4 \%$ & $6,1 \%$ \\
\hline Multimedia & $14,6 \%$ & $1,2 \%$ & $15,9 \%$ \\
\hline Material impreso (libros, revistas) & $13,4 \%$ & $1,2 \%$ & $14,6 \%$ \\
\hline Texto escolar & $9,8 \%$ & $6,1 \%$ & $15,9 \%$ \\
\hline Material creado por el estudiantado & $4,9 \%$ & $0,0 \%$ & $4,9 \%$ \\
\hline Sitios Web & $1,2 \%$ & $0.0 \%$ & $1,2 \%$ \\
\hline Tecnología/Software & $1,2 \%$ & $8,5 \%$ & $9,8 \%$ \\
\hline \multicolumn{4}{|c|}{ Adquisición, compresión y comunicación del contenido* } \\
\hline Escuchando & $18,7 \%$ & $18,7 \%$ & $37,4 \%$ \\
\hline Leyendo & $10,1 \%$ & $2,9 \%$ & $12,9 \%$ \\
\hline Hablando & $16,5 \%$ & $9,4 \%$ & $25,9 \%$ \\
\hline Escribiendo & $16,5 \%$ & $7,2 \%$ & $23,7 \%$ \\
\hline
\end{tabular}

*Valor de $\mathrm{p}<.05 ;{ }^{* *}$ valor de $\mathrm{p}<.001$

Nota: Elaboración propia. 


\section{Discusión de resultados}

En este artículo se analizan los resultados obtenidos a través de un diagnóstico de las prácticas pedagógicas observadas en aulas de Lenguaje y Comunicación y Matemática lideradas por docentes de tercero medio en liceos participantes en el programa PACE UCSC. Si bien parte de los resultados obtenidos en este estudio evidencia las fortalezas y debilidades con respecto al desempeño pedagógico del equipo docente participante, la información recopilada durante el proceso del diagnóstico de prácticas pedagógicas fue un insumo fundamental para establecer planes de acción individualizados y definidos conforme a las necesidades detectadas por cada docente participante en el programa de acompañamiento pedagógico asociado al programa PACE. No obstante, al sistematizar la información de todo el equipo docente participante y considerando el contenido de los cursos observados, se encuentran patrones comunes con respecto a las prácticas y recursos pedagógicos comúnmente utilizados en las aulas participantes.

Siguiendo con las reformas educacionales dirigidas hacia la instalación de un sistema de aseguramiento de la calidad de la enseñanza, el Ministerio de Educación chileno publica los Estándares Indicativos de Desempeño para los Establecimientos Educacionales y sus Sostenedores. Este documento contiene un "conjunto de referentes que constituyen un marco orientador para la evaluación de los procesos de gestión", de los principales actores que conforman un establecimiento educativo (Mineduc, República de Chile, 2014, p. 5). Así, el proceso de enseñanza-aprendizaje en el aula se encuentra definido por seis estándares (tres de los cuales fueron considerados en este estudio). Cada estándar indicativo de desempeño establece una rúbrica que detalla los "criterios que describen los procedimientos, prácticas, cualidades o logros más relevantes", definiendo las siguientes categorías: débil, incipiente, satisfactorio y avanzado dependiendo de la valoración en la implementación de este mismo (Mineduc, República de Chile, 2014, p. 34). Adicionalmente, se describen los medios de diagnóstico que pueden ser utilizados para evaluar el nivel de desarrollo de cada estándar. En el caso de las prácticas de aula asociadas al proceso de enseñanza y aprendizaje, se incluye la observación de clases como una estrategia válida para su diagnóstico. En esta investigación se incluyen los resultados de observaciones de aula que son atribuibles a los elementos definidos en el desarrollo de estándares 5.1, 5.2 y 5.3 del proceso de enseñanza y aprendizaje en el aula.

Considerando los criterios establecidos con respecto al desarrollo en el aula de cada estándar, es posible indicar lo siguiente:

Estándar 5.1: El personal docente observado acostumbra a mantener una planificación acorde con los objetivos de aprendizaje establecidos en el currículo chileno para cada curso observado. Claramente esta es una práctica institucionalizada. De esta manera, es posible considerar que la implementación se encuentra en un nivel satisfactorio conforme a los indicadores de desempeño establecidos para este estándar. La pauta de observación consideró consultar al estudiantado de la clase sobre el objetivo de la lección con el propósito de conocer 
doi: http://dx.doi.org/10.15359/ree.23-2.8

URL: http://www.una.ac.cr/educare

CORREO: educare@una.cr

si estos lo comprendían. Si bien, este no es un indicador definido para este estándar, es recomendable introducir cada clase explicando al estudiantado el objetivo y las expectativas de aprendizaje esperadas, o bien verbalizarlo en algún momento de la clase de acuerdo con la organización de esta (deductivo o inductivo). Al establecer el objetivo al inicio de la clase, se permite que el estudiantado logre establecer un propósito sobre lo que van a aprender, lo que confiere responsabilidad al estudiantado con respecto a los aprendizajes esperados al finalizar la clase (Marzano, 2011).

Estándar 5.2: El profesorado conduce las clases con claridad, rigurosidad conceptual, dinamismo e interés. Se observó que existen diferencias entre las estrategias mayormente empleadas por los docentes y las docentes de Lenguaje y Comunicación y de Matemática. En este sentido, la tendencia observada es que los docentes y las docentes de Lenguaje y Comunicación tienden a utilizar una mayor variedad de estrategias de enseñanza en comparación con sus pares de Matemática, aunque en general los recursos pedagógicos utilizados no representan una gran diversidad con respecto a las alternativas mayormente utilizadas en prácticas de enseñanza efectivas. Adicionalmente, se observa que el equipo docente tiende al diseño de clases que presentan un bajo desafío cognitivo por parte del estudiantado (conocimiento, comprensión y aplicación). De acuerdo con los criterios indicativos de desempeño, se aprecia un desarrollo débil en la implementación de este estándar. En estudios relacionados con escuelas con baja eficacia en el aprendizaje del estudiantado se ha observado que se caracterizan por la utilización de estrategias didácticas limitadas a la reproducción de contenidos, donde usualmente el equipo docente establece las preguntas entregando pocas posibilidades al estudiantado para interactuar con el contenido de la clase (Murillo et al., 2016). En este tipo de escuelas se suele observar que privilegian la enseñanza reproductiva y memorística, no utilizan recursos didácticos motivadores, hay ausencia de atención a la diversidad y mala gestión del tiempo (HernándezCastilla, Murillo y Martínez-Garrido, 2014). Es posible identificar muchos de estos elementos de las escuelas ineficaces, en los establecimientos educacionales participantes en este estudio.

Estándar 5.3: El profesorado utiliza estrategias efectivas de enseñanza-aprendizaje en el aula. Este estándar fue valorado a partir del tipo de actividades que desarrolla el estudiantado de la clase. Al igual que lo registrado en el estándar 5.2, se evidencian diferencias entre las prácticas comúnmente utilizadas por los docentes y las docentes de Lenguaje y Comunicación y Matemática, donde se distingue una mayor variedad de materiales de instrucción utilizado en las clases de Lenguaje y Comunicación observadas. En ambas asignaturas, se privilegia la enseñanza a toda la clase o el trabajo individual. En este sentido, es deseable promover el trabajo colaborativo en el aula, lo que se encuentra alineado con las actuales políticas educacionales chilenas y una gran cantidad de investigaciones que identifican al trabajo entre pares como una buena práctica en el aula de clase (Lin, Justice, Paul y Mashburn, 2016). Por último, es importante destacar la manera en que el estudiantado interactúa con los contenidos de las clases observadas. Al analizar los porcentajes totales con respecto a la adquisición, 
comprensión y comunicación del contenido, se observa una relativa utilización homogénea de las cuatro estrategias registradas para este ítem. Sin embargo, destacan las diferencias entre las estrategias utilizadas en Lenguaje y Comunicación y Matemática, donde en este último caso el estudiantado participa mayormente escuchando las instrucciones del docente durante la clase. Igualmente, llama la atención que la lectura sea el recurso menos utilizado en clases. Conforme a las pautas de valoración para este estándar, se aprecia un desarrollo incipiente en la implementación de estrategias efectivas de enseñanza-aprendizaje en el aula.

\section{Conclusiones}

Las observaciones de salas de clase tienen altos niveles de validez aparente, porque evalúan prácticas de enseñanza que el propio profesorado puede observar. Para aquel personal docente que se esfuerza por convertirse en mejores profesionales en la práctica, esta información puede proporcionar retroalimentación formativa oportuna y accionable (Cohen y Goldhaber, 2016). Así, los resultados producto de las visitas al aula como parte de la implementación de la estrategia PAAD del programa PACE UCSC fueron utilizados para reflexionar con respecto a la práctica pedagógica del equipo docente en el aula. Se entregó una retroalimentación detallada a cada docente participante para luego acordar de manera conjunta, un plan de acción de asociado a sus requerimientos y necesidades identificadas durante la fase de diagnóstico de prácticas pedagógicas. Así, las observaciones de aula deben ser vistas como una efectiva herramienta que permite facilitar procesos mejoramiento docente. Donde, el involucramiento del profesorado en la observación y recolección de información sobre prácticas docentes, genera un conocimiento compartido entre colegas con un significativo efecto sobre sus própias prácticas en el aula.

Los sistemas de aseguramiento de la calidad de la enseñanza son relativamente nuevos para el sistema educativo chileno. Sin duda, todavía queda mucho trabajo para que el personal docente se encuentre cada vez más familiarizado con los estándares indicativos de desempeño publicados en el 2014. En este sentido, existe consenso en que las características del profesorado y el desempeño de sus estudiantes se encuentran asociados (Bravo, Falck, González, Manzi y Peirano, 2008; Lara, Mizala y Repetto, 2010). Recientemente, la utilización de estrategias de activación cognitiva y la instrucción orientada en la investigación reportaron un efecto positivo sobre el desempeño académico del estudiantado en distintos sistemas educativos (Blazar, 2015; Caro et al., 2016). No obstante, es importante reconocer que el resultado académico del estudiantado puede responder a múltiples factores, muchos de los cuales no los maneja el equipo docente. De esta forma, las características asociadas a la docencia efectiva no deberían ser evaluadas netamente por los resultados académicos de sus estudiantes, más bien el centro debe ponerse en las prácticas pedagógicas desplegadas por los docentes y las docentes en el aula, las que se deberían traducir en una mejora académica del estudiantado dentro de sus propios contextos de enseñanza. 
doi: http://dx.doi.org/10.15359/ree.23-2.8

URL: http://www.una.ac.cr/educare

CORREO: educare@una.cr

En un futuro se espera continuar realizando observaciones de este tipo, donde el profesorado pueda tomar un rol más activo en el proceso de revisión de prácticas pedagógicas. En este sentido, la mayoría de los instrumentos de evaluación que involucran observación de aula requieren múltiples observaciones con un mínimo de tiempo de duración y con una separación entre cada observación de semanas a meses para maximizar la posibilidad de una estimación estable y adecuada de la práctica docente (Hill, Charalambous y Kraft, 2012). Sería deseable establecer diferentes eventos de observación de aula a lo largo de un año académico, donde los mismos cuerpos docentes puedan participar en las observaciones de sus pares. Cabe señalar que las instancias de observación de clase no deben investirse de evaluación punitiva para con el profesorado, sino que se espera que se constituya en una estrategia para recabar datos clave que propicien acciones de mejoramiento de las prácticas docentes, lo que vendría a proyectarse en una enseñanza de calidad de acuerdo con los estándares propuestos por e Mineduc. De esta forma, desde la estrategia PAAD del PACE UCSC, se espera contribuir a la constitución de comunidades profesionales de aprendizaje en las que todos los entes involucrados formen parte del proceso de observación, desde su concepción hasta su valoración.

En este estudio, el análisis de las observaciones de las prácticas pedagógicas se hizo desde el paradigma que asume el modelo educativo que propone el MINEDUC mediante las bases curriculares y los Estándares Indicativos de Desempeño. Es así que la caracterización de las prácticas pedagógicas que aplican los profesores y profesoras de los Liceos PACE dan cuenta de los desafios para alcanzar aprendizajes de calidad. Entre estos están, el poco uso de recursos adecuados, la marcada tendencia por mantener al estudiantado escuchando las explicaciones e instrucciones de su docente y su escasa participación activa al interior del aula. El valor intrínseco del estudio es que se presentan evidencias empíricas que permiten respaldar la necesidad de mejorar las practicas pedagógicas que usan los profesores y profesoras que atienden a niños, niñas y jóvenes pertenecientes a los sectores con mayor vulnerabilidad social.

\section{Limitaciones}

El estudio formó parte del programa de acompañamiento a las prácticas docentes involucradas en la implementación del programa operativo del PACE UCSC 2015. Hubiese sido deseable realizar una segunda observación durante el año académico, para poder contrastar los resultados junto con el personal docente participante y reflexionar sobre eventuales cambios en las practicas seleccionadas y trabajas en el plan de acción acordado con cada docente de las áreas de lenguaje y matemáticas. Esta situación no fue posible de concretar, debido al movimiento docente que se tradujo en casi dos meses de paro, lo que acortó el año académico. Por otro lado, si bien cada observador se rigió por un protocolo de observación que establecía los parámetros generales para valorar las categorías definidas, los resultados registrados pueden están influenciados por la subjetividad del sujeto observador. Esto podría 
ser evitado al incorporar un segundo personaje observador simultáneo que permita calibrar los registros en las pautas de evaluación. En este caso, no se logró implementar este diseño por motivos logísticos y para evitar causar la sensación de intervención en el aula. Una muy buena opción para desarrollar estos registros podría ser filmar cada clase, lo que también supone un alto grado de intervención al equipo docente de aula. Por último, se debe tener claro que la caracterización de prácticas pedagógicas es un proceso complejo. En este estudio, se utiliza como referencia el Marco para la Buena Enseñanza de Chile para caracterizar el desempeño del profesorado, sin embargo no se debe desconocer la existencia de otras miradas con respecto a lo que se entiende por desempeño docente efectivo dentro de las que caben las prácticas pedagógicas desde la afectividad y emocionalidad.

\section{Financiamiento}

El desarrollo de la investigación se enmarca dentro de la implementación del programa PACE financiado por el Ministerio de Educción de Chile y ejecutado por la Universidad Católica de la Santísima Concepción. Los instrumentos de recogida de información, análisis de datos, desarrollo de ideas y conclusiones forman parte de la propuesta técnica desarrollada por el equipo ejecutor de la propuesta y no han sido revisadas ni aprobados por alguna agencia gubernamental que financie la propuesta.

\section{Agradecimientos}

La autoría de este artículo agradece la participación de los 58 docentes de Lenguaje y Comunicación y Matemática que formaron parte activa en la implementación de la estrategia de preparación y acompañamiento docente del programa PACE. También, agradece las valiosas aportaciones de la Dra. Alejandra Nocetti, las que ayudaron a mejorar las versiones finales del manuscrito del artículo.

\section{Referencias}

Avanzini, G. (2004). Capacitación en estrategias y técnicas didácticas. Monterrey, México: Instituto Tecnológico y de Estudios Superiores de Monterrey.

Blazar, D. (2015). Effective teaching in elementary mathematics : Identifying classroom practices that support student achievement. Economics of Education Review, 48, 16-29. https://doi. org/10.1016/j.econedurev.2015.05.005

Bravo, D., Falck, D., González, R., Manzi, J. y Peirano, C. (2008). La relación entre la evaluación docentey el rendimiento de los alumnos: Evidencia para el caso de Chile. Recuperado de http://eoepsabi. educa.aragon.es/descargas/H Recursos/h 1 Psicol_Educacion/h 1.4.Eval desemp docente/1.5.Relacion evaluac doc rendim.pdf 
doi: http://dx.doi.org/10.15359/ree.23-2.8

URL: http://www.una.ac.cr/educare

CORREO: educare@una.cr

Caro, D. H., Lenkeit, J. y Kyriakides, L. (2016). Teaching strategies and differential effectiveness across learning contexts : Evidence from PISA 2012. Studies in Educational Evaluation, 49, 30-41. doi: https://doi.org/10.1016/j.stueduc.2016.03.005

Castro-Pérez, M. y Morales-Ramírez, M. E. (2015). Los ambientes de aula que promueven el aprendizaje, desde la perspectiva de los niños y niñas escolares. Revista Electrónica Educare, 19(3), 1-32. http://dx.doi.org/10.15359/ree.19-3.11

Cohen, J. y Goldhaber, D. (2016). Building a More Complete Understanding of Teacher Evaluation Using Classroom Observations. Educational Researcher, 45(6), 378-387. doi: https://doi. org/10.3102/0013189X16659442

Goldhaber, D. (2008). Teachers matter, but effective teacher quality policies are elusive. En H. F. Ladd y E. B. Fiske (Eds.), Handbook of research in education finance and policy (pp. 146-165). New York, NY: Routledge.

González-Weil, C., Cortéz, M., Bravo, P., Ibaceta, Y., Cuevas, K., Quiñones, P., ... Abarca, A. (2012). La indagación científica como enfoque pedagógico: Estudio sobre las prácticas innovadoras de docentes de ciencia en EM (Región de Valparaíso). Estudios Pedagógicos, 38(2), 85-102. doi: https://doi.org/10.4067/S0718-07052012000200006

Gore, J. M., Smith, M., Bowe, J., Ellis, H. R., Lloyd, A. y Lubans, D. R. (2015). Quality teaching rounds as a professional development intervention for enhancing the quality of teaching: Rationale and study protocol for a cluster randomised controlled trial. International Journal of Educational Research, 74, 82-95. doi: https://doi.org/10.1016/j.ijer.2015.08.002

Hanushek, E. A. (1997). Assessing the effects of school resources on student performance: An update. Educational Evaluation and Policy Analysis, 19(2), 141-164. doi: https://doi. org/10.3102/01623737019002141

Hargrove, R. (2011). Fostering creativity in the design studio: A framework towards effective pedagogical practices. Art, Design y Communication in Higher Education, 10(1), 7-31. doi: https://doi.org/10.1386/adch.10.1.7 1

Hernández-Castilla, R., Murillo, F. J., y Martínez-Garrido, C. (2014). Factores de ineficacia escolar. REICE. Revista Iberoamericana sobre Calidad, Eficacia y Cambio, 12(1), 103-118. Recuperado de https://revistas.uam.es/index.php/reice/article/view/2867/3084

Hill, H. C., Charalambous, C. Y. y Kraft, M. A. (2012). When rater reliability is not enough: Teacher observation systems and a case for the generalizability study. Educational Researcher, 41(2), 56-64. doi: https://doi.org/10.3102/0013189X12437203 
Kim, Y.y Silver, R.E.(2016). Provoking reflective thinking in post observation conversations. Journal of Teacher Education, 67(3), 203-219. doi: https://doi.org/10.1177/0022487116637120

Kri, F., Gil, F. J., González, M. y Lamatta, C. (2013). Ranking de notas como predictor del éxito en educación superior. Estudio de caso USACH Informe final. Santiago de Chile; CNED. Recuperado de http://www.cned.cl/proyecto-de-investigacion/ranking-de-notas-comopredictor-del-exito-en-educacion-superior-estudio-de

Lara, B., Mizala, A. y Repetto, A. (2010). Una mirada a la efectividad de los profesores en Chile. Estudios Publicos, 120, 147-182.

Latorre, M. (2004). Aportes para el analisis de las racionalidades presentes en las prácticas pedagogicas. Estudios Pedagógicos, 30, 75-91. doi: https://doi.org/10.4067/S0718$\underline{07052004000100005}$

Liang, J. (2015). Live video classroom observation: An effective approach to reducing reactivity in collecting observational information for teacher professional development. Journal of Education for Teaching, 41(3), 235-253. doi: https://doi.org/10.1080/02607476.2015.1045314

Lin, T.-J., Justice, L. M., Paul, N., y Mashburn, A. J. (2016). Peer interaction in rural preschool classrooms: Contributions of children's learning-related behaviors, language and literacy skills, and problem behaviors. Early Childhood Research Quarterly, 37, 106-117. doi: https:// doi.org/10.1016/j.ecresq.2016.04.001

Martinez, F., Taut, S. y Schaaf, K. (2016). Classroom observation for evaluating and improving teaching: An international perspective. Studies in Educational Evaluation, 49, 15-29. doi: https://doi.org/10.1016/j.stueduc.2016.03.002

Marzano, R. J. (2011). The art and science of teaching. Alexandria,VA: ASCD.

McLeskey, J., Waldron, N., Spooner, F. y Algozzine, B. (Eds.). (2014). Handbook of effective inclusive schools: Research and practice. New York, NY: Routledge. doi: https://doi. org/10.4324/9780203102930

Ministerio de Educación, República de Chile. (2008). Marco para la buena enseñanza. Recuperado de portales.mineduc.cl/usuarios/cpeip/File/Documentos\%202011/MBE2008.pdf

Ministerio de Educación, República de Chile. (2014). Estándares indicativos de desempeño para establecimientos educacionales y sus sostenedores. Recuperado de http://archivos. agenciaeducacion.cl/documentos-web/Estandares_Indicativos_de_Desempeno.pdf

Ministerio de Educación, República de Chile. (2015). Fundamentos del PACE. Elementos que fundamentan este programa y su etapa piloto. Recuperado de www.uchile.cl/documentos/ fundamentos-del-pace 1105495 3633.pdf 
doi: http://dx.doi.org/10.15359/ree.23-2.8

URL: http://www.una.ac.cr/educare

CORREO: educare@una.cr

Murillo, F.J.,Hernández-Castilla, R.,y Martínez-Garrido,C.(2016).¿Quéocurreen lasaulas dondelos niños y niñas no aprenden? Estudio cualitativo de aulas ineficaces en Iberoamérica. Perfiles Educativos, 38(151), 55-70. doi: https://doi.org/10.22201/iisue.24486167e.2016.151.54886

Rockoff, J. E. (2004). The impact of individual teachers on student achievement: Evidence from panel data. The American Economic Review, 94(2), 247-252. doi: https://doi. org/10.1257/0002828041302244

Steinberg, M. P. y Garrett, R. (2016). Classroom composition and measured teacher performance: What do teacher observation scores really measure? Educational Evaluation and Policy Analysis, 38(2), 293-317. doi: https://doi.org/10.3102/0162373715616249

The Charles A. Dana Center. (2011). Classroom walkthrough for continuous improvement: Strengthening teaching and learning. Austin, TX: Autor. 